conclude that the preclinical animal models used are impressive in their prediction of human MTD (maximum tolerated dose) and DLT (dose limiting toxicities).

This detailed preclinical data can then be used to optimize the design of the clinical trials. This account explains in some detail how a clear understanding of a drugs pharmacology can lead to improved clinical use. A greater understanding of these pharmacokinetic-pharmacodynamic relationships has led to optimization of the administration and delivery of new agents in Phase I trials.

A major new development in clinical trial design is the ability to measure the distribution and, to a certain extent, the pharmacodynamic effect of these new agents in a non-invasive manner. PET (positron emission tomography) has a whole chapter dedicated to the technique and it is clear the power of the technique will influence the design of future clinical trials. PET can now be used for pharmacokinetic analysis with examples, for 5-FU and temozolomide given. It can be used for pharmacodynamic studies, for example drug receptor interactions and cellular proliferation, even detecting apoptotic cells in situ.
The concluding chapter discusses trials of the newly emerging mechanistic drugs. It describes trial design for the compounds such as copolymers, intended to take advantage of the leaky vasculature of tumours, and signal transduction inhibitors where it is now necessary to build in pharmacodynamic endpoints, showing target inhibition/activation, into clinical trials alongside the currently established pharmacokinetic monitoring.

In summary, this is a really extensive and comprehensive book on the drug development process. It has detailed chapters on potential targets, the drug design process, the drug screening process and the design of future clinical trials to cope with these new mechanistic based drugs. There is very little overlap between chapters and all are written by experts in the field. Hundreds of useful references are included for those wanting to go further. My one and only problem was finding the colour prints tucked away at the back. Maybe they were too expensive to include in the text, but I found them eventually. Congratulations to the editors as this must have been quite a task. Very good value for $\$ 100$ and essential reading for both the scientist and Ph.D. student. Definitely one for the library to stock.

Paul Loadman Cancer Research Unit University of Bradford, UK

\title{
Corrigendum
}

\section{Neuroblastoma in monozygotic twins - a case of probable twin to twin metastasis}

\author{
J Anderson, H Kempski, L Hill, D Rampling, T Gordon and A Michalski
}

\author{
DOI: 10.1038/sj/bjc/6600308 www.bjcancer.com \\ (C) 2002 Cancer Research UK
}

Correction to: British Journal of Cancer (2001) 85, 493-496. DOI: $10.1054 /$ bjoc.2001.1979

The authors would like to acknowledge the Research into Childhood Cancer charity (Gibraltar) (RICC) for funding for the above project. 